\title{
Comparison of several closure approximations for evaluating the thermoelastic properties of an injection molded short-fiber composite
}

\author{
Delphine Dray, Pierre Gilormini, Gilles Régnier* \\ Laborato ire d’Ingénierie des Matériaux (CNRS UMR 8006), \\ ENSAM Paris, 151 Bvd de l’Hôpital, 75013 Paris, France
}

\begin{abstract}
The accurate prediction of both the elastic properties and the thermal expansion coefficients is very important for the precise simulation of such processes as injection molding of short-fiber polymer-matrix composites. In this work, a two-step homogenization procedure is applied and compared with experimental values obtained on a polyarylamide/glass fiber composite for a broad range of temperatures. It is observed that the stiffness averaging version of the model surpasses the compliance averaging variant, especially when it is combined with a precise evaluation of the fourth-order orientation tensor. It is also demonstrated that the orthotropic closure approximations are significantly better than previous ones (linear, quadratic, and hybrid) and than a very recent one. Among the orthotropic closure approximations, the fitted ones lead to acceptable results, which are very close to those obtained with the experimentally measured fourth-order orientation tensor.
\end{abstract}

Keywords: (A) Short-fiber composites, (B) Thermoelastic properties, (C) Elastic properties, (C) Orientation tensors, (E) Injection molding

${ }^{*}$ Correspond ing author. Tel:33 144 2463 05, Fax :33 1442463 82, gilles.regnier@paris.ensam.fr 


\section{Introduction}

Short-fiber reinforced polymer-matrix composites constitute an important class of technical materials, because of their technological and economical interests. In particular, such composites with non-aligned reinforcements are of considerable importance because of their good thermoelastic properties. Even if both the matrix and the fibers are isotropic, the composite is usually anisotropic because of the non random orientations of the fibers: it is stiffer and stronger along the direction of dominant orientation, for instance. In the present paper, both the elastic and thermal properties of a polyarylamide/glass fiber composite, obtained by injection molding, are estimated and measured.

Several theories $([1,2]$ for instance) proceed in two steps to predict the overall thermoelastic properties of such materials: first, the properties of a unidirectional composite are estimated, and then an orientation averaging procedure is applied over all directions. For the first step, it has been shown by Tucker and Liang [3] that, among a set of available theories, the Mori and Tanaka model [4, 5] gives satisfactory results when compared with finite element simulations. This is consistent with the previous comparisons with experimental results performed by Peyroux [6], for instance. In this paper, emphas is is put on the orientation averaging stage. Materials with isotropic distributions of fiber orientations [5, $7,8]$ and/or planar isotropic distributions [9] have been considered much less often than unidirectional composites, and even fewer studies have focused on general orientation distributions $[10,11,12]$. The orientation distribution function, which describes the probability of finding fibers with a given orientation in the specimen, depends on two angular variables and is uneasy to manipulate in commercial codes for the simulation of injection molding. This has been used by Pierard et al. [13] though, but it has also been proposed to rather define suitable orientation tensors, which depend on a small number of variables. Advani and Tucker [14], for instance, used orientation tensors that had been introduced by 
Hand [15]. The second and the fourth-order orientation tensors are needed to apply most micromechanical models, but the fourth-order one is unavailable usually and, therefore, several closure approximations have been proposed. This is the type of approach that is evaluated in the present paper.

The paper is organized as follows. In Section 2, the two-level homogenization technique used to predict the elastic properties from the distribution of fiber orientations is described, and the procedure to deduce the thermal expansion coefficients is detailed. In section 3, experimental determinations of fiber orientation and thermoelastic properties of both matrix and composite are presented. In Section 4, the theory is applied to a polyarylamide/glass fiber composite with a distribution of fiber orientations that is typical of injection molding. Several approximation closures are implemented, and the results are compared with original experimental data.

\section{Theory}

\subsection{Elastic properties}

As mentioned in the Introduction, the effective elastic properties of a unidirectional composite containing up to $20 \%$ by volume of short fibers can be evaluated accurately with the Mori-Tanaka model. Detailed derivations can be found in [3], for instance, and only the final expression needs being given here:

$$
\mathbf{C}^{U D}=\mathbf{C}^{m}+c_{f}\left(\mathbf{C}^{f}-\mathbf{C}^{m}\right): \mathbf{A}^{M T}
$$

where letters $f$ and $m$ refer to the fibers and to the matrix, respectively, with stiffness tensors $\mathbf{C}^{f}$ and $\mathbf{C}^{m}$. Perfect bonding is assumed between the matrix and the fibers (with a volume fraction $c_{f}$ ), which are axially symmetric. The strain concentration tensor in the fibers $\mathbf{A}^{M T}$ that the model deduces is obtained from the Eshelby tensor [16] $\mathbf{E}$ for a prolate spheroid having the same aspect ratio as the fibers: 


$$
\mathbf{A}^{M T}=\mathbf{A}:\left[\left(1-c_{f}\right) \mathbf{I}+c_{f} \mathbf{A}\right]^{-1} \quad \text { with } \quad \mathbf{A}=\left[\mathbf{I}+\mathbf{E}: \mathbf{S}^{m}:\left(\mathbf{C}^{f}-\mathbf{C}^{m}\right)\right]^{-1}
$$

where $\mathbf{I}$ is the fourth-order identity tensor and $\mathbf{S}^{m}=\left(\mathbf{C}^{m}\right)^{-1}$ denotes the compliance of the matrix material. The components of the Eshelby tensor, which depend on the fiber aspect ratio and on the matrix elastic constants, can be computed from the expressions given by Mura [17]. An important property of the Mori-Tanaka model is that the dual approach in terms of compliances, leading to

$$
\mathbf{S}^{U D}=\mathbf{S}^{m}+c_{f}\left(\mathbf{S}^{f}-\mathbf{S}^{m}\right): \mathbf{B}^{M T} \quad \text { with } \quad \mathbf{B}^{M T}=\mathbf{C}^{f}: \mathbf{A}^{M T}: \mathbf{S}^{m}
$$

where $\mathbf{B}^{M T}$ is the stress concentration tensor in the fibers, is such that $\mathbf{S}^{U D}$ is the inverse of $\mathbf{C}^{U D}$

When a distribution of fiber orientations is present in a composite, the method of Advani and Tucker [14] can be used, where the properties of an auxiliary unidirectional composite are weighted by the orientation distribution function. In terms of the elevation and azimuthal angles, $\theta$ and $\varphi$, the effective stiffness of the composite then writes as

$$
\mathbf{C}=\int_{\Omega} \mathbf{C}^{U D}(\theta, \varphi) \psi(\theta, \varphi) \mathrm{d} \Omega
$$

where $\psi(\theta, \varphi)$ denotes the orientation distribution function and $\Omega$ is the unit sphere, with $\int_{\Omega} \psi(\theta, \varphi) \mathrm{d} \Omega=1$ and $\mathrm{d} \Omega=\sin \theta \mathrm{d} \theta \mathrm{d} \varphi$. The stiffness of the auxiliary unidirectional composite $\mathbf{C}^{U D}(\theta, \varphi)$ is obtained from (1) with the symmetry axis defined by the angles $\theta$ and $\varphi$, by taking $c_{f}$ equal to the total volume fraction of fibers in the misoriented composite. The latter condition may lead to a quite stiff auxiliary unidirectional composite, but th is is balanced by the weighting procedure. This method, proposed by Dunn et al. [10] and recently applied by Pierard et al. [13], requires the probability density function to be specified. Although it can be determined numerically from flow-induced fiber rotations for simple flow conditions, it is very expensive to compute for three-dimensional flows. Its experimental 
determination is possible, but this requires a lot of effort and time. Therefore, simplified descriptions of the orientation distribution are preferred, such as using orientation tensors defined by the second and fourth moments of the orientation distribution function:

$$
a_{i j}=\int_{\Omega} p_{i} p_{j} \psi(\theta, \varphi) \mathrm{d} \Omega \quad \text { and } \quad a_{i j k l}=\int_{\Omega} p_{i} p_{j} p_{k} p_{l} \psi(\theta, \varphi) \mathrm{d} \Omega
$$

where $\mathbf{p}(\theta, \varphi)$ is a unit vector parallel to a fiber direction, with components

$$
p_{1}=\sin \theta \cos \varphi \quad p_{2}=\sin \theta \sin \varphi \quad p_{3}=\cos \theta .
$$

The expressions in (5) clearly show that $a_{i j}=a_{i i}$ and, similarly, that any permutation of the four subscripts keeps $a_{i j k l}$ constant. Moreover, $p_{1}^{2}+p_{2}^{2}+p_{3}^{2}=1$ implies that

$$
a_{i i}=1 \quad a_{i j i j}=1 \quad \text { and } \quad a_{i j i j}=1
$$

(with summation over repeated subscripts, as everywhere in this paper). As a result, there are only 5 independent $a_{i j}$ components and only 13 independent $a_{i j k l}$ components. Moreover, the following relation is obtained easily between the components of the two tensors:

$$
a_{i j 11}+a_{i j 22}+a_{i j 33}=a_{i j} .
$$

Isotropic fibers and matrix are considered in this work, and therefore the auxiliary unidirectional composite is transversely isotropic and can be written as [2]:

$$
\begin{aligned}
C_{i j k l}^{U D}(\theta, \varphi)= & C_{1} p_{i} p_{j} p_{k} p_{l}+C_{2}\left(p_{i} p_{j} \delta_{k l}+p_{k} p_{l} \delta_{i j}\right)+C_{3}\left(p_{i} p_{k} \delta_{j l}+p_{i} p_{l} \delta_{j k}\right. \\
& \left.+p_{j} p_{l} \delta_{i k}+p_{j} p_{k} \delta_{i l}\right)+C_{4} \delta_{i j} \delta_{k l}+C_{5}\left(\delta_{i k} \delta_{j l}+\delta_{i l} \delta_{j k}\right)
\end{aligned}
$$

where $\delta_{i j}$ is the Kronecker symbol and the five constants $C_{1}, \ldots, C_{5}$ are related to the standard components of the stiffness tensor (with symmetry around axis 1):

$$
\begin{array}{cc}
C_{1}=C_{3333}^{U D}-2 C_{3322}^{U D}+C_{2211}^{U D}-4 C_{3232}^{U D}+2 C_{2121}^{U D} & C_{2}=C_{3322}^{U D}-C_{2211}^{U D} \\
C_{3}=C_{3232}^{U D}-C_{2121}^{U D} \quad C_{4}=C_{2211}^{U D} & C_{5}=C_{2121}^{U D}
\end{array} .
$$

Consequently, the orientation av eraging (4) leads to:

$$
\begin{aligned}
C_{i j k l}= & C_{1} a_{i j k l}+C_{2}\left(a_{i j} \delta_{k l}+a_{k l} \delta_{i j}\right)+C_{3}\left(a_{i k} \delta_{j l}+a_{i l} \delta_{j k}+a_{j l} \delta_{i k}+a_{j k} \delta_{i l}\right) \\
& +C_{4} \delta_{i j} \delta_{k l}+C_{5}\left(\delta_{i k} \delta_{j l}+\delta_{i l} \delta_{j k}\right)
\end{aligned}
$$


Alternatively, an averaging of the compliance tensor of the auxiliary unidirectional composite, defined by five constants $S_{1}, \ldots, S_{5}$, leads to :

$$
\begin{aligned}
S_{i j k l}= & S_{1} a_{i j k l}+S_{2}\left(a_{i j} \delta_{k l}+a_{k l} \delta_{i j}\right)+S_{3}\left(a_{i k} \delta_{j l}+a_{i l} \delta_{j k}+a_{j l} \delta_{i k}+a_{j k} \delta_{i l}\right) \\
& +S_{4} \delta_{i j} \delta_{k l}+S_{5}\left(\delta_{i k} \delta_{j l}+\delta_{i l} \delta_{j k}\right)
\end{aligned}
$$

but, in spite of $\mathbf{S}^{U D}$ being the inverse of $\mathbf{C}^{U D}$, this effective compliance $\mathbf{S}$ is not the inverse of the effective stiffness $\mathbf{C}$ obtained above. This is a limitation of the present two-step approach. Many authors $[2,14,18]$ prefer stiffness averaging to compliance averaging, because of a better agreement with experimental elastic constants, but without discussing the effect on thermal properties. In the present work, both the elastic and thermal properties predicted for the composite are considered, and they are compared with experimental results in Section 4.

\subsection{Closure approximations}

Another drawback of the orientation averaging method is that the fourth-order tensor $a_{i j k l}$ is required to evaluate the effective stiffness (or compliance) tensor, whereas flow simulation codes usually store the second-order tensor $a_{i j}$ only. Several ways of relating these two tensors have been suggested, such as the linear, quadratic, and hybrid first generation closure hypotheses. They are given respectively by:

$$
a_{i j k l}^{L}=-\frac{1}{35}\left(\delta_{i j} \delta_{k l}+\delta_{i k} \delta_{j l}+\delta_{i l} \delta_{j k}\right)+\frac{1}{7}\left(a_{i j} \delta_{k l}+a_{i k} \delta_{j l}+a_{i l} \delta_{j k}+a_{k l} \delta_{i j}+a_{j l} \delta_{i k}+a_{j k} \delta_{i l}\right)
$$

which is exact for a completely isotropic distribution of fiber orientations,

$$
a_{i j k l}^{Q}=a_{i j} a_{k l}
$$

which is exact for aligned fibers, and

$$
a_{i j k l}^{H}=(1-f) a_{i j k l}^{L}+f a_{i j k l}^{Q}
$$


which is an intermediate between the quadratic and the linear closures weighted by a coefficient $0 \leq f \leq 1$. Two expressions for the weighting function $f$ in terms of the tensor $a_{i j}$ have been suggested by Advani and Tucker [14, 19], such that $f=1$ for perfectly aligned fibers and $f=0$ for a completely isotropic distribution:

$$
f=\frac{3}{2} a_{i j} a_{i j}-\frac{1}{2} \quad \text { or } \quad f=1-27 \operatorname{det}\left(a_{i j}\right)
$$

where $\operatorname{det}\left(a_{i j}\right)$ denotes the determinant of tensor $a_{i j}$. In the sequel, type A and type B hybrid closures will refer to using respectively the left and right part of (16). For the special cases where the fiber distribution is planar, two-dimensional variants of (16) have also been proposed [19]. Since the experimental fiber distribution considered is not strictly planar, as shown below, emphasis will be put on the three-dimensional versions of the closure hypotheses in the present work.

Noting that fiber orientation distributions frequently include a strong 1D or 2D component, Doghri and Tinel [20] have very recently proposed a new closure approximation by weighting the $1 \mathrm{D}, 2 \mathrm{D}$ and $3 \mathrm{D}$ components of a general fiber distribution:

$$
A=\alpha_{1} A^{1 D}+\alpha_{2} A^{2 D}+\alpha_{3} A^{3 D}, \quad \alpha_{1}=\frac{a_{1}-a_{2}}{a_{1}}, \alpha_{2}=\frac{a_{2}-a_{3}}{a_{1}}, \alpha_{1}=\frac{a_{3}}{a_{1}}
$$

where $a_{\mathrm{i}}$ denote the eigenvalues of the orientation tensor $a_{i j}\left(a_{1>} a_{2}>a_{3}\right), A^{1 \mathrm{D}}$ is the quadratic closure, $A^{2 \mathrm{D}}$ and $A^{3 \mathrm{D}}$ being deduced from the hybrid closure. Much earlier, Cintra and Tucker [21] had defined fourth-order orientation tensors that use the principal axes of the secondorder tensor $a_{i j}$ to define the three planes of an orthotropic symmetry. Such fourth-order tensors $a_{i j k l}$ can be written as $A_{m n}$ in the contracted $6 \times 6$ notation that is usual in the theory of elasticity. When expressed in the principal coordinate axes, an orthotropic fourth-order tensor has nine independent components, but once full symmetry and normalization conditions (7) are applied, the number of independent components reduces to 3 . Since the sum of $a_{1}, a_{2}$ and $a_{3}$ equals 1 , defining an orthotropic closure approximation reduces simply to choosing three 
scalar functions $f$, such that $a_{i i i i}=f_{i}\left(a_{1}, a_{2}\right)$ (no sum over $i$ ). Cintra and Tucker [21] used second-degree polynomials:

$$
a_{i i i i}=C_{i 1}+C_{i 2} a_{1}+C_{i 3} a_{1}^{2}+C_{i 4} a_{2}+C_{i 5} a_{2}^{2}+C_{i 6} a_{1} a_{2}
$$

where $C$ is a $3 \times 6$ matrix of coefficients defined either by using specific orientation distributions (1D, random 2D or 3D, leading to the smooth version of their approach), or by fitting on distributions obtained for a set of flow fields (fitted version). Later, Chung and Kwon [22] extended the set of flow fields and obtained another fit for the second-order polynomials; they also proposed using third-degree polynomial functions. At this point, it may be mentioned that the linear closure approximation is orthotropic and can be written in the form of (18):

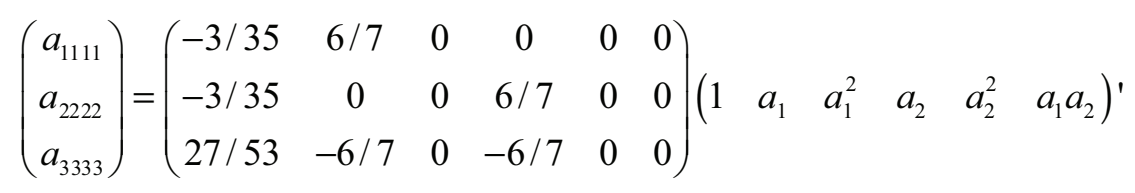

The $3 \times 6$ matrices pertaining to the smooth and fitted Cintra-Tucker approaches can be found in [21], and another one is given by Chung and Kwon [22], using their improved fitting procedure. The quadratic closure is not orthotropic, and therefore this applies also to the hybrid closure and to the one proposed Doghri and Tinel. The natural closure approximation proposed by Verleye et al. [23] has orthotropic symmetry, but the three independent components of the fourth-order tensor are defined as polymonial functions of the secondorder tensor invariants. As Cintra and Tucker have got very similar results for their fitted closure and the natural one on several flow fields, the latter closure approximation was not tested in the present work. It can be also noted that the natural closure approximation is tedious to compute.

Consequently, several evaluations of the elastic properties of the composite will be obtained, according to whether the stiffnesses or the compliances are averaged and depending on the closure approximation used: linear, quadratic, hybrid of type A or B, Doghri-Tinel 
closure, smooth and fitted variants of the Cintra-Tucker approach, and the second-order polynomial fitted by Chung and Kwon.

\subsection{Thermal expansion}

In thermoelastic materials, the stresses are related to the total strain and to the temperature change by

$$
\boldsymbol{\sigma}=\mathbf{C}:(\boldsymbol{\varepsilon}-\boldsymbol{\alpha} \Delta T)=\mathbf{C}: \boldsymbol{\varepsilon}-\mathbf{\kappa} \Delta T
$$

where $\boldsymbol{\alpha}$ and $\boldsymbol{\kappa}$ are symmetric second-order tensors that define the thermal expansion and the thermal stress, respectively. This suggests strongly that the thermal properties of a composite are closely related to its elasticity. This is especially true for a short-fiber composite contain ing two phases only, where the relation obtained by Levin [21] applies:

$$
\boldsymbol{\alpha}=\left(\mathbf{S}^{f}-\mathbf{S}\right):\left(\mathbf{S}^{f}-\mathbf{S}^{m}\right)^{-1}: \boldsymbol{\alpha}^{m}+\left(\mathbf{S}^{m}-\mathbf{S}\right):\left(\mathbf{S}^{m}-\mathbf{S}^{f}\right)^{-1}: \boldsymbol{\alpha}^{f} .
$$

It means that the thermal expansion can be evaluated directly from an estimation of the

effective compliance $\mathbf{S}$ tensor. This remarkable result is obtained from the general expression $\boldsymbol{\alpha}=\left(1-c_{f}\right) \boldsymbol{\alpha}^{m}: \mathbf{B}^{m}+c_{f} \boldsymbol{\alpha}^{f}: \mathbf{B}^{f}$ where the stress localization ten sors $\mathbf{B}^{m}$ and

$\mathbf{B}^{f}$ in the two phases are eliminated by using $\mathbf{I}=\left(1-c_{f}\right) \mathbf{B}^{m}+c_{f} \mathbf{B}^{f}$ and

$\mathbf{S}=\left(1-c_{f}\right) \mathbf{S}^{m}: \mathbf{B}^{m}+c_{f} \mathbf{S}^{f}: \mathbf{B}^{f}$. Similarly, elimination of the strain localization tensors $\mathbf{A}^{m}$ and $\mathbf{A}^{f}$ between $\boldsymbol{\kappa}=\left(1-c_{f}\right) \boldsymbol{\kappa}^{m}: \mathbf{A}^{m}+c_{f} \mathbf{\kappa}^{f}: \mathbf{A}^{f}, \mathbf{I}=\left(1-c_{f}\right) \mathbf{A}^{m}+c_{f} \mathbf{A}^{f}$ and $\mathbf{C}=\left(1-c_{f}\right) \mathbf{C}^{m}: \mathbf{A}^{m}+c_{f} \mathbf{C}^{f}: \mathbf{A}^{f}$ implies that the thermal stress can be estimated directly from the effective stiffness tensor as:

$$
\boldsymbol{\kappa}=\left(\mathbf{C}^{f}-\mathbf{C}\right):\left(\mathbf{C}^{f}-\mathbf{C}^{m}\right)^{-1}: \boldsymbol{\kappa}^{m}+\left(\mathbf{C}^{m}-\mathbf{C}\right):\left(\mathbf{C}^{m}-\mathbf{C}^{f}\right)^{-1}: \boldsymbol{\kappa}^{f}
$$

Usually, (22) is not used because it is equivalent to (21) when $\mathbf{\kappa}=\mathbf{C}: \boldsymbol{\alpha}$ (which is expected from (20)) and $\mathbf{C}=\mathbf{S}^{-1}$ are used, but the latter relation does not apply to the model considered in this work, which leads to effective stiffness and compliance tensors that are not inverse to 
each other because of the orientation averaging procedure. As a result, (21) on the one hand, and (22) combined with $\boldsymbol{\alpha}=\mathbf{C}^{-1}: \boldsymbol{\kappa}$ on the other hand, provide two different evaluations of the thermal expansion.

The fact that the effective compliance tensor is obtained here from an averaging procedure has another important consequence in the special case that we consider, where the thermoelastic properties of both the matrix and the fibers are isotropic. In these conditions, the effective compliance tensor $\mathbf{S}$, which is anisotropic for a general orientation distribution of the fibers, is contracted in (21) with the isotropic second-order tensor $\left(\mathbf{S}^{f}-\mathbf{S}^{m}\right)^{-1}:\left(\boldsymbol{\alpha}^{f}-\boldsymbol{\alpha}^{m}\right)$. Since any isotropic second-order tensor is proportional to the second-order identity tensor $\delta_{i j}$, the term containing $\mathbf{S}$ in (21) will be proportional to

$$
\begin{aligned}
S_{i j k l} \delta_{k l}= & S_{1} a_{i j k l} \delta_{k l}+S_{2}\left(a_{i j} \delta_{k l}+a_{k l} \delta_{i j}\right) \delta_{k l}+S_{3}\left(a_{i k} \delta_{j l}+a_{i l} \delta_{j k}+a_{j l} \delta_{i k}+a_{j k} \delta_{i l}\right) \delta_{k l} \\
& +S_{4} \delta_{i j} \delta_{k l} \delta_{k l}+S_{5}\left(\delta_{i k} \delta_{j l}+\delta_{i l} \delta_{j k}\right) \delta_{k l} \\
= & \left(S_{1}+3 S_{2}+4 S_{3}\right) a_{i j}+\left(S_{2}+3 S_{4}+2 S_{5}\right) \delta_{i j}
\end{aligned}
$$

according to (13), and using (8) to replace $a_{i j k k}$ by $a_{i j}$. Consequently, the thermal expansion obtained will depend on the second-order orientation tensor only, and will be independent of the closure approximation considered. Similar arguments apply to the thermal stress, but using $\boldsymbol{\alpha}=\mathbf{C}^{-1}: \boldsymbol{\kappa}$ subsequently to get thermal expansion will reintroduce an influence of the closure approximation through its effect on $\mathbf{C}$.

Another method for obtaining the thermal properties, similar to what has been done in the previous section for the elastic properties, consists in performing the orientation averaging directly on the thermal properties of the auxiliary unidirectional composite. Applying Levin's formula to this composite, one obtains:

$$
\boldsymbol{\alpha}^{U D}=\left(\mathbf{S}^{f}-\mathbf{S}^{U D}\right):\left(\mathbf{S}^{f}-\mathbf{S}^{m}\right)^{-1}: \boldsymbol{\alpha}^{m}+\left(\mathbf{S}^{m}-\mathbf{S}^{U D}\right):\left(\mathbf{S}^{m}-\mathbf{S}^{f}\right)^{-1}: \boldsymbol{\alpha}^{f}
$$

and

$$
\boldsymbol{\kappa}^{U D}=\left(\mathbf{C}^{f}-\mathbf{C}^{U D}\right):\left(\mathbf{C}^{f}-\mathbf{C}^{m}\right)^{-1}: \boldsymbol{\kappa}^{m}+\left(\mathbf{C}^{m}-\mathbf{C}^{U D}\right):\left(\mathbf{C}^{m}-\mathbf{C}^{f}\right)^{-1}: \mathbf{\kappa}^{f}
$$


which are equivalent through $\mathbf{\kappa}^{U D}=\mathbf{C}^{U D}: \boldsymbol{\alpha}^{U D}$ (recall that $\mathbf{C}^{U D}=\left(\mathbf{S}^{U D}\right)^{-1}$ ). Since (24) is linear with respect to $\mathbf{S}^{U D}$, the assumption that both phases are isotropic implies that averaging $\boldsymbol{\alpha}^{U D}$ comes down to averaging $\mathbf{S}^{U D}$ only in (24), what leads exactly to (21). The same considerations apply to (25), which gives (22). Therefore, this method does not provide new evaluations of the thermal properties in the present case.

Consequently, several evaluations of the effective thermal expansion coefficients are obtained: one is independent of the closure approximation and is deduced from compliance averaging, whereas stiffness averaging leads to different variants for each closure approximation used. They are compared with experimental values in Section 4.

\section{Experimental}

A short-fiber composite (IXEF 1002 supplied by Solvay) has been analyzed in this study. It was made of a polyarylamide (semi-crystalline aromatic polyamide) matrix containing 16.5 $\%$ (volume fraction) of glass fibers with an aspect ratio equal to 25 . Both constituents were isotropic, with an influence of temperature on the Young modulus, Poisson's ratio and thermal expansion coefficient of the matrix as shown in Fig. 1, whereas these parameters were assumed to be temperature-independent in the fibers, and equal to $74 \mathrm{GPa}, 0.25$ and $510^{-6} \mathrm{C}^{-1}$, respectively. The matrix Young modulus was measured by tensile and dynamic torsion tests; the expansion coefficient by dilatometry tests and the Poisson's ratio was deduced from the compressibility modulus $K$ measurement as $v=(3-E / K) / 6$.

$60 \times 60 \times 1 \mathrm{~mm}^{3}$ plates of this polyarylamide/glass fiber composite have been mold injected through a $0.8 \mathrm{~mm}$-thick end-fan gate to provide a parallel flow front. Orientation distributions and moduli have been measured in the center of the plates. Since the thickness of the plate is small, the fiber distribution does not change significantly through the thickness (for instance, $a_{11}$ in the flow direction does not vary more than 15\%) and no skin-core distribution appears. 
The Young moduli (at room temperature and at $120 \mathrm{C}$ ) have been measured with tensile tests, using a uniaxial extensometer; and the thermal expansion coefficients (for a whole range of temperatures) with dilatometry tests. Because the specimens were very thin, the Young moduli were measured along the injection flow (axis 1) and the transverse direction (axis 2) only, whereas the thermal expansion could be obtained also through the plate thickness (axis $3)$.

The fiber orientation tensors were measured accurately by analyzing a series of images obtained with a scanning electron microscope, following a procedure that reduced the possible artifacts by using inclined polished cuts (this will be reported in a separate paper). The components of the measured $a_{i j}$ tensor deduced from the observation of a large number of fibers are:

$$
a_{i j}=\left(\begin{array}{lll}
0.793 & 0.016 & 0.053 \\
0.016 & 0.179 & 0.006 \\
0.053 & 0.006 & 0.028
\end{array}\right)
$$

where it can be observed that the distribution is dominantly in the injection plane (the $a_{11}$ and $a_{22}$ terms are large) but is not strictly planar, and that many fibers are parallel to the injection flow ( $a_{11}$ is the largest component). These $a_{i j}$ values will be used in all the numerical applications that follow. The fourth-order orientation tensor also was deduced from the direct observation of the fibers, and all its components can be obtained from the following set of 15 values:

$$
\begin{aligned}
& a_{1111}=0.694 \quad a_{1122}=0.080 \quad a_{1133}=0.019 \quad a_{1112}=0.018 \quad a_{1123}=0.004 \quad a_{1131}=0.042 \\
& a_{2222}=0.094 \quad a_{2233}=0.005 \quad a_{2212}=-0.003 \quad a_{2223}=0.002 \quad a_{2231}=0.006 \\
& a_{3333}=0.004 \quad a_{3312}=0.001 \quad a_{3323}=0.000 \quad a_{3331}=0.005
\end{aligned}
$$

It can be checked that the two sums $a_{i i j j}$ and $a_{i j i j}$ are equal to 1 (which does lead to 13 independent components), and that (8) is satisfied. This experimental fourth-order orientation 
tensor allows computing the elastic and thermal properties without using any closure hypothesis, and this will be applied in the discussion below.

\section{Results and discussion}

First, the use of the various closure approximations for predicting the elastic properties is analyzed. Table 1 presents the experimental Young moduli measured along the flow axis $\left(E_{l}\right)$ of the composite and along the transverse direction $\left(E_{2}\right)$ at room temperature and at $120 \mathrm{C}$. These values are significantly higher than for pure matrix (Fig. 1), especially along the direction of preferred fiber orientation, which illustrates the effect of reinforcements. It is worth noting that considering a low and a high temperature provides an interesting test of the homogenization model: the same microstructure (i.e. set of fiber orientations) is considered with two phase 'contrasts', since the elastic constants of the fibers do not change, whereas the polymer matrix Young modulus decreases for a temperature higher than its glass transition temperature; therefore the contrast is higher. As a result, the ratio between $E_{1}$ and $E_{2}$ increases at high temperature.

In Table 1, these experimental values are compared with the results given by stiffness and compliance averagings, using the experimental second-order orientation tensor and the linear, quadratic, and hybrid closure approximations mentioned above, whereas Table 2 compares to more elaborate closure approximations and to using the experimentally measured fourth-order orientation tensor, with stiffness averaging. It can be observed first in Table 1 that the predictions are better when the contrast between the phases is lower, i.e. at low temperature, especially for $E_{2}$ (with a precision of a few percent). It also appears that stiffness averaging provides better results than compliance averaging almost systematically, especially for the $E_{l}$ modulus at both temperatures. Among the various closure hypotheses applied with stiffness averaging in Table 1, the linear approximation is slightly better than others globally, although 
it tends to underestimate the moduli (down to $-19 \%$ for $E_{1}$ at high temperature). It may also be noted that the difference between the two hybrid variants is quite small, and that their predictions are not always bounded by the linear and quadratic models (although each component of the fourth-order orientation tensor is), which illustrates the complex interactions between constituents that are involved in a homogenization procedure.

Table 2 demonstrates thate the orthotropic closure approximations, combined with stiffness averaging, lead to very good results. The closure of Doghri and Tinel [20], which is not orthotropic, compares poorly with the experiments, since it leads to the largest overestimates of $E_{l}$ among all models. It can also be observed that the Chung and Kwon closure [22] gives slightly larger moduli than the fitted version of the Cintra and Tucker closure [21], which is very close to the values deduced from the experimental fourth-order orientation tensor, and close to the measured moduli. The smooth version of the Cintra and Tucker closure leads to higher, and therefore less satisfactory, values.

It can be concluded from Tables 1 and 2 that the linear closure and the fitted Cintra-Tucker closure give equivalently good predictions of the elastic moduli, with the former (which is a very simple special case of an orthotropic closure) tending to underestimate the experimental results and the latter giving overestimates. The thermal expansion coefficients will provide a further test of the theories. These coefficients were obtained along three axes defined by the injection flow. The latter are not exactly material symmetry axes (recall that $a_{12}, a_{23}$ and $a_{31}$ are not zero in (26)): $\alpha_{11}, \alpha_{22}$ and $\alpha_{33}$ are not the principal values of the $\boldsymbol{\alpha}$ tensor, and small nonzero $\alpha_{12}, \alpha_{23}$ and $\alpha_{31}$ components were measured, that will not be reported here. These experimental results, for a whole range of temperatures, are first compared in Fig. 2 with the thermal expansion coefficients $\alpha_{11}, \alpha_{22}$ and $\alpha_{33}$ deduced from compliance averaging. The latter approach does not make any difference between the various closure approximations: these predictions only use the experimental second-order orientation tensor $a_{i j}$ and the whole 
curves shown in Fig. 1. It can be noted in Fig. 2 that the predictions are not very good: an increase of $\alpha_{11}$ with temperature is predicted rather than the observed decrease, and the difference with the experimental results keeps increasing with temperature for $\alpha_{11}, \alpha_{22}$ and $\alpha_{33}$. Some additional flexibility for a better agreement could be expected from the role played by the closure approximation in the stiffness averaging approach, and Fig. 3 presents the results given by the linear, quadratic, and hybrid closure hypotheses. It is interesting to note first that temperature enhances the differences between the models, which can thus be discriminated. A decrease of $\alpha_{11}$ with temperature is predicted now, but it is overestimated and unacceptable negative values are obtained at high temperatures, whichever of these closure approximations is considered, with the linear variant being worst. By contrast, this variant keeps reasonably close to the $\alpha_{22}$ experimental results (although the trend is wrong at high temperature, with a decrease), which are increasingly overestimated by the other closure approximations. All four closure hypotheses behave in a comparable way for predicting the $\alpha_{33}$ thermal coefficient and the results are all acceptable, with better results for the hybrid variant of type B. The difference between the two hybrid closure approximations is more significant for thermal expansion coefficients than it was for Young moduli: both types of hybrid models keep between the linear and quadratic approximations, with type A staying very close to the quadratic approximation and type B being closer to the linear variant. Therefore, the combination of stiffness averaging and linear closure approximation that appeared to be the best for predicting the Young moduli fails in giving reasonable values of the thermal expansion, essentially because of largely negative $\alpha_{11}$ values at high temperatures. Finally, it may be mentioned that the planar version of the linear closure approximation and the corresponding hybrid variants (as defined in [14] and [19]) were also applied, since $a_{33}$ is small, by merely discarding the suitable components of $a_{i j}$ and $a_{i j k l}$ (and 
amplifying $a_{11}$ and $a_{22}$ such that $a_{11}+a_{22}=1$ ), but this did not modify the above conclusions significantly.

The results given by more recent closure approximations are compared to the experimental measures in Fig. 4, where the predictions deduced directly from the experimental fourth-order orientation tensor are also reported. First, the deficiency of the Doghri-Tinel closure procedure that was already suggested above by the elastic moduli is clearly confirmed here by the thermal expansion coefficients. Although both are orthotropic and use the same number of nonzero coefficients, as mentioned in Section 2, the smooth Cintra-Tucker closure gives better results than the linear closure approximation, but it is less satisfactory than other orthotropic closures for the $\alpha_{11}$ thermal expansion coefficient. Although they give very similar results, the fitted Cintra-Tucker closure is slightly closer than the Chung-Kwon closure to the direct use of the experimental fourth-order orientation tensor. This suggests that the fitted Cintra-Tucker closure hypothesis is excellent and that the refinements added by Chung and Kwon are not really useful, at least for the cases we studied. Fig. $4 \mathrm{c}$ shows a very close similitude between four approaches for the $\alpha_{33}$ coefficient: the smooth Cintra-Tucker closure of and the Chung-Kwon closure on the one hand, the fitted Cintra-Tucker closure and the use of the experimental orientation tensor on the other hand, cannot be distinguished; moreover, these two sets are close to each other. It can be observed that the trends are correct when temperature increases for all thermal expansion coefficients, and that no negative value is obtained, but that too large (resp. small) values are predicted for $\alpha_{22}$ (resp. $\left.\alpha_{33}\right)$ at high temperatures. This may be due to the complex flow-induced crystalline microstructure of the polymer matrix, which cannot be considered as fully isotropic. The dilatometric behavior of the pure injection molded matrix is transversely isotropic, with a larger expansion coefficient along the thickness direction. The fitted Cintra-Tucker closure approximation is nevertheless the best among all the variants studied here, since it performs as 
well as the experimental fourth-order orientation tensor. Moreover, its good predictions confirm that the simple two-step homogenization procedure that averages the stiffness predicted by the Mori-Tanaka model for a unidirectional composite is reasonable.

\section{Conclusions}

(i) The comparison with experimental values of both the elastic and thermal properties of a short-fiber composite provides a selective procedure to test predictive models.

(ii) The two-step homogenization procedure that applies orientation averaging to an auxiliary unidirectional composite with the same fiber content as the misoriented composite is able to predict the elastic and thermal properties of a short-fiber composite accurately.

(iii) A good agreement has been observed with experimental results obtained on an injection molded polyarylamide/glass fiber composite at low and high temperatures, what means for a broad range of contrasts between the properties of the two phases.

(iv) The stiffness averaging version of the model surpasses the compliance averaging variant, in particular for the thermal expansion coefficients because it allows more flexibility through the role of the fourth-order orientation tensor.

(v) The linear, quadratic, and hybrid closure approximations lead to unacceptable results; the linear approximation is found to be the best as far as the elastic properties are considered, but it gives bad results for the thermal expansion along the injection flow direction. The closure approximation proposed recently by Doghri and Tinel does not compare well with our experimental results for both elastic moduli and thermal expansion.

(vi) The fitted Cintra and Tucker closure is the best among all the approximations studied here, since it performs as well as the experimental fourth-order orientation tensor. Its combination with a simple stiffness averaging procedure leads to good predictions for elastic moduli and thermal expansion. 


\section{Acknowledgements}

The authors acknowledge LEGRAND, MOLDFLOW and SOLVAY companies for their financial support. They wish to thank H. Alglave, J. Correa, D. Delaunay, R. Fulchiron, S. Karpp-Pfordt, P. Kennedy, M. Laplanche, R. Le-Goff, V. Leo, J.M. Rossignol, R. Zheng for the enriching discussions during all the FISH (FIber filled polymers: injection and SHrinkage) project.

\section{References}

[1] Lielens G, Pirotte P, Couniot A, Dupret F, Keunings R. Prediction of thermo-mechanical properties for compression moulded composites. Composites Part A 1998;29A:63-70.

[2] Mlekusch B. Thermoelastic properties of short-fibre-reinforced thermoplastics. Comp Sci Technol 1999; 59:911-923.

[3] Tucker III CL, Liang E. Stiffness predictions for unidirectional short-fiber composites: Review and evaluation. Comp Sci Technol 1999; 59:655-671.

[4] Mori T, Tanaka K. Average stress in matrix and average elastic energy of materials with misfitting inclusions. Acta Metall 1973;21:571-574.

[5] Benveniste Y. A new approach to the application of Mori-Tanaka's theory in composite materials. Mech Mater 1987; $6: 147-157$.

[6] Peyroux R. Caractéristiques thermoélastiques de matériaux composites à fibres courtes.

Ph. D. Thesis, Université Montpellier II Sciences et Techniques du Languedoc, 1990.

[7] Taya M, Dunn ML, Derby B, Walker J. Thermal residual stress in a two-dimensional inplane misoriented short fiber composite. Appl Mech Rev 1990; 43:S294-S303.

[8] Chen T, Dvorak GJ, Benveniste Y. Mori-Tanaka estimates of the overall elastic moduli of certain composite materials. J Appl Mech 1992; 59:539-546. 
[9] Tandon GP, Weng GJ. Average stress in the matrix and effective moduli of randomly oriented composites. Comp Sci Technol 1986; 27:111-132.

[10] Dunn ML, Ledbetter H, Heyliger PL, Choi CS. Elastic constants of textured short-fiber composites. J Mech Phys Solids 1996; 44:1509-1541.

[11] Dunn ML, Ledbetter H. Micromechanically-based acoustic characterization of the fiber orientation distribution function of morphologically textured short-fiber composites: prediction of thermomechanical and physical properties. Mater Sci Engng 2000; A285:56-61. [12] Takao Y, Chou TW, Taya M. Effective longitudinal Young's modulus of misoriented short fiber composites. J Appl Mech 1982; 49:536-540.

[13] Pierard O, Friebel C, Doghri I. Mean-field homogenization of multi-phase thermo-elastic composites: a general framework and its validation. Comp Sci Technol 2004; 64:1587-1603. [14] Advani SG, Tucker III CL. The use of tensors to describe and predict fiber orientation in short fiber composites. J Rheol 1987; 31:751-784.

[15] Hand GL. A theory of anisotropic fluids. J Fluid Mech 1962; 13:33-46.

[16] Eshelby JD. The determination of the elastic field of an ellipsoidal inclusion, and related problems. Proc R Soc Lond 1957; A241:376-396.

[17] Mura T. Micromechanics of defects in solids. Martinus Nijhoff Publishers, Boston, 1987.

[18] Gupta M, Wang KK. Fiber orientation and mechanical properties of short-fiberreinforced injection-molded composites: simulated and experimental results. Polymer Comp $1993 ; 14: 367-382$.

[19] Advani SG, Tucker III CL. Closure approximations for three-dimensional structural tensors. J. Rheol. 1990; 34: 367-386. 
[20] Doghri I, Tinel L. Micromechanics of inelastic composites with misaligned inclusions: numerical treatment of orientation. Comput. Methods Appl. Mech. Engng. 2006; 195:13871406.

[21] Cintra JS, Tucker III CL. Orthotropic closure approximations for flow-induced fiber orientation. J. Rheol. 1995; 39:1095-1121.

[22] Chung DH, Kwon TH. Improved model of orthotropic closure approximation for flow induced fiber orientation. Polymer Composites 2001;22:636-649.

[23] Verleye V, Couniot A, Dupret F. Numerical prediction of fiber orientation in complex composite injection-molded parts. ASME -MD 1994; 49:265-279.

[24] Levin VM. Thermal expansion coefficients of heterogeneous materials. Mekhanika Tverdogo Tela 1967; 2:88-94. English translation in Mech Solids 1967;2:58-61. 


\section{Figure Captions}

Figure 1: Experimental values of the matrix Young modulus (a), Poisson's ratio (b) and thermal expansion coefficient (c), versus temperature.

Figure 2: Thermal expansion coefficients along the three axes of the composite: experimental values (symbols) and predictions given by the compliance averaging procedure.

Figure 3: Thermal expansion coefficients along the three axes of the composite: experimental values (symbols) and predictions given by the stiffness averaging procedure using the linear (unbroken line), quadratic (dotted line), and hybrid (type A: long dashes, type B: short dashes) closure approximations.

Figure 4: Same as Figure 3, but the experimental values (symbols) are now compared to the predictions given by the stiffness averaging procedure using either the experimental fourthorder orientation tensor (unbroken line), or the closure approximations proposed by Cintra and Tucker (smooth closure: mixed dashes, fitted closure: long dashes), Chung and Kwon (short dashes), Doghri and Tinel (dotted line). 


\section{Table Captions}

Table 1: Young moduli of the composite along the flow direction $\left(E_{l}\right)$ and along the transverse direction $\left(E_{2}\right)$, in GPa: experimental values and predictions given by either stiffness averaging or compliance averaging, using four closure approximations (linear, quadratic, and hybrid of type A or type B).

Table 2: Young moduli of the composite along the flow direction $\left(E_{l}\right)$ and along the transverse direction $\left(E_{2}\right)$, in GPa: experimental values and predictions given by stiffness averaging using either the experimental fourth-order orientation ten sor or the closure approximations proposed by Cintra and Tucker (smooth and fitted versions: CTs and CTf), Chung and Kwon (CK), Doghri and Tinel (DT). 
stiffness averaging compliance averaging

exp. lin. quad. hyb.A hyb.B lin. quad. hyb.A hyb.B

\begin{tabular}{|c|c|c|c|c|c|c|c|c|c|c|}
\hline \multirow{2}{*}{$\begin{array}{l}\text { room } \\
\text { temp. }\end{array}$} & $\mathrm{E}_{1}$ & 11.80 & 10.58 & 11.05 & 11.02 & 10.86 & 9.13 & 9.59 & 9.54 & 9.35 \\
\hline & $\mathrm{E}_{2}$ & 7.22 & 7.40 & 6.91 & 6.96 & 7.16 & 7.15 & 6.89 & 6.91 & 7.02 \\
\hline \multirow{2}{*}{$120 \mathrm{C}$} & $\mathrm{E}_{1}$ & 3.74 & 3.03 & 3.15 & 3.19 & 3.25 & 1.36 & 1.51 & 1.49 & 1.43 \\
\hline & $\mathrm{E}_{2}$ & 1.26 & 1.14 & 0.94 & 0.96 & 1.06 & 0.98 & 0.91 & 0.92 & 0.94 \\
\hline
\end{tabular}




\begin{tabular}{cccccccc} 
& & exp. & exp. $\mathrm{a}_{\mathrm{ijk} l}$ & CTs & CTf & CK & DT \\
\hline \multirow{2}{*}{$\begin{array}{l}\text { room } \\
\text { temp. }\end{array}$} & $E_{1}$ & 11.80 & 12.18 & 12.91 & 12.28 & 12.38 & 14.23 \\
\cline { 2 - 8 } & & & & & & & \\
\hline
\end{tabular}


Figure 1

(a)

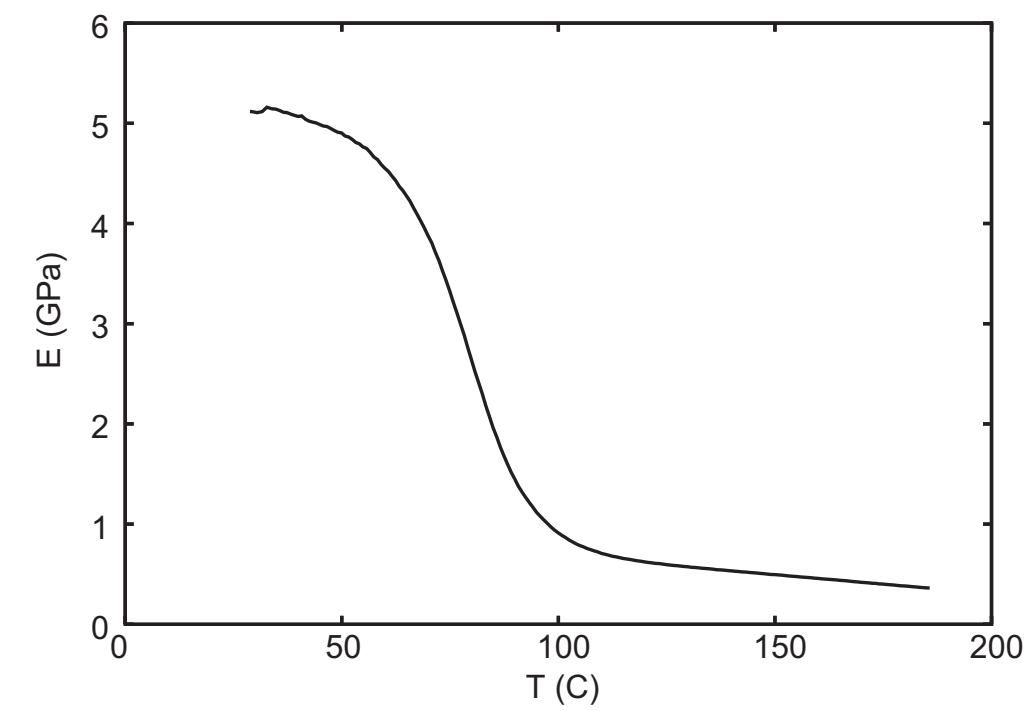

(b)
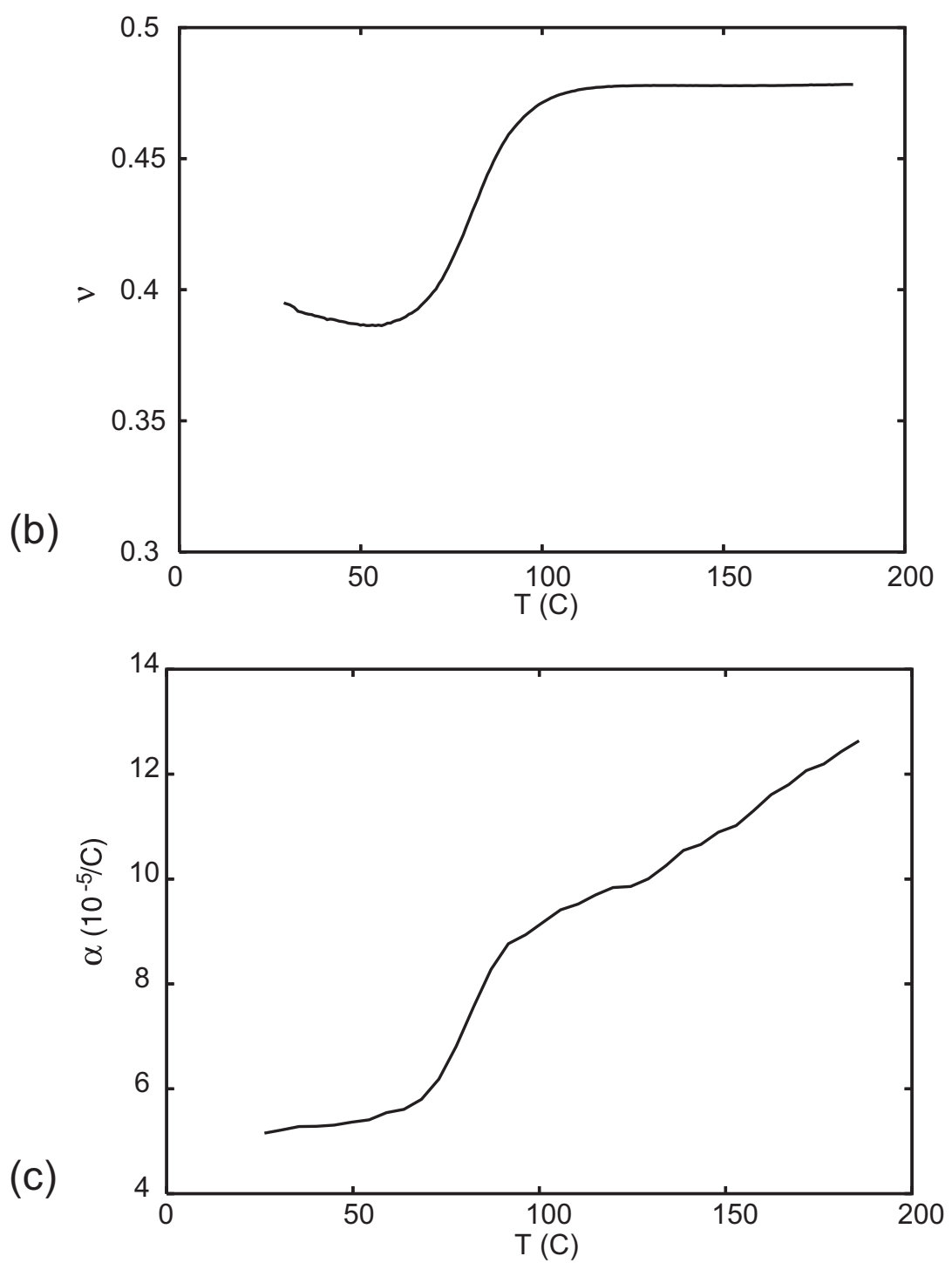
Figure 2

(a)

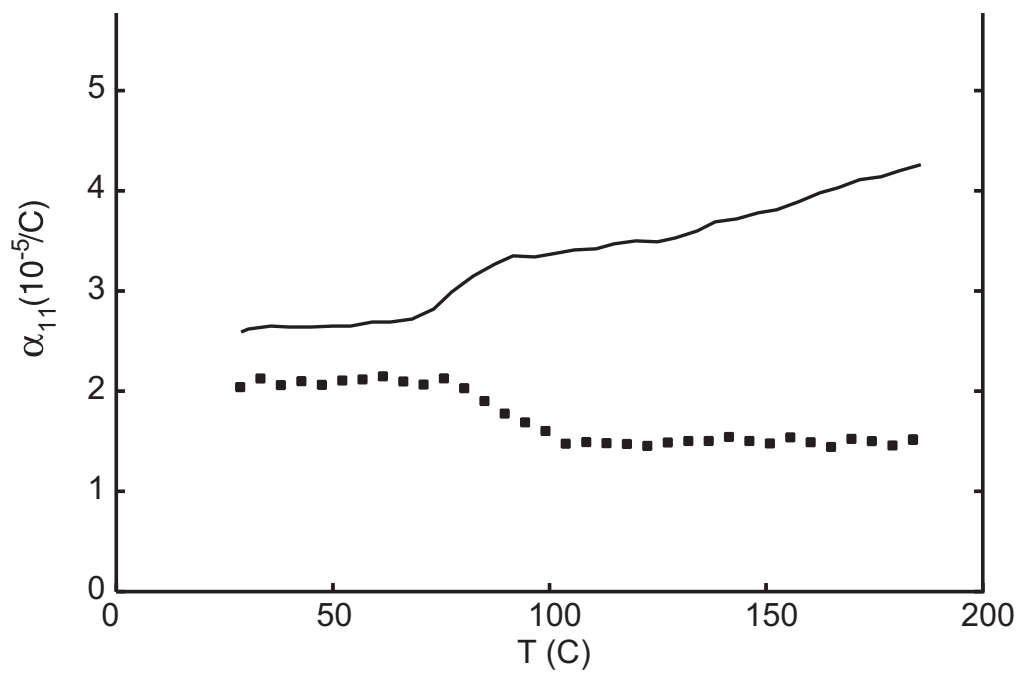

(b)
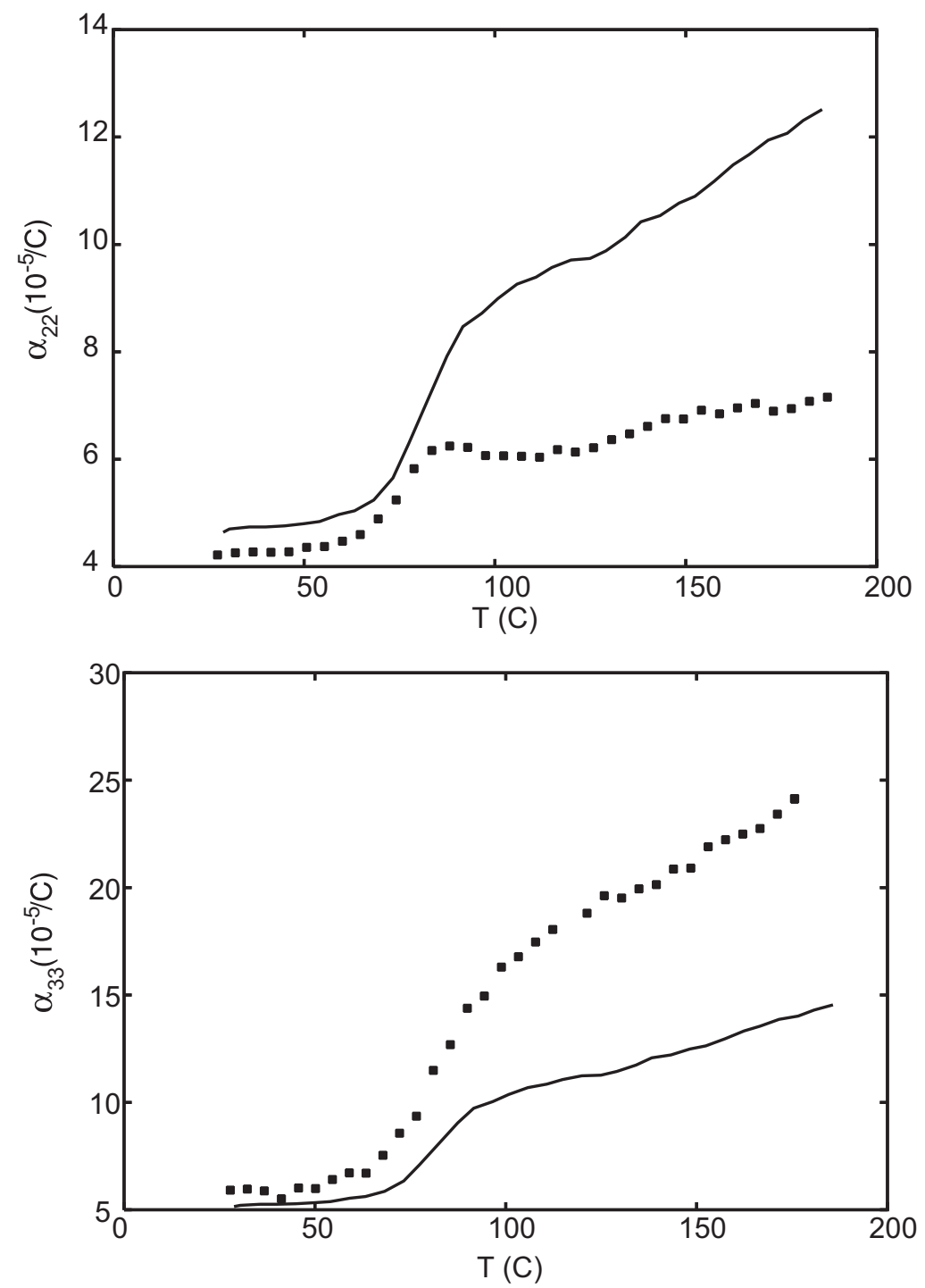
Figure 3

(a)

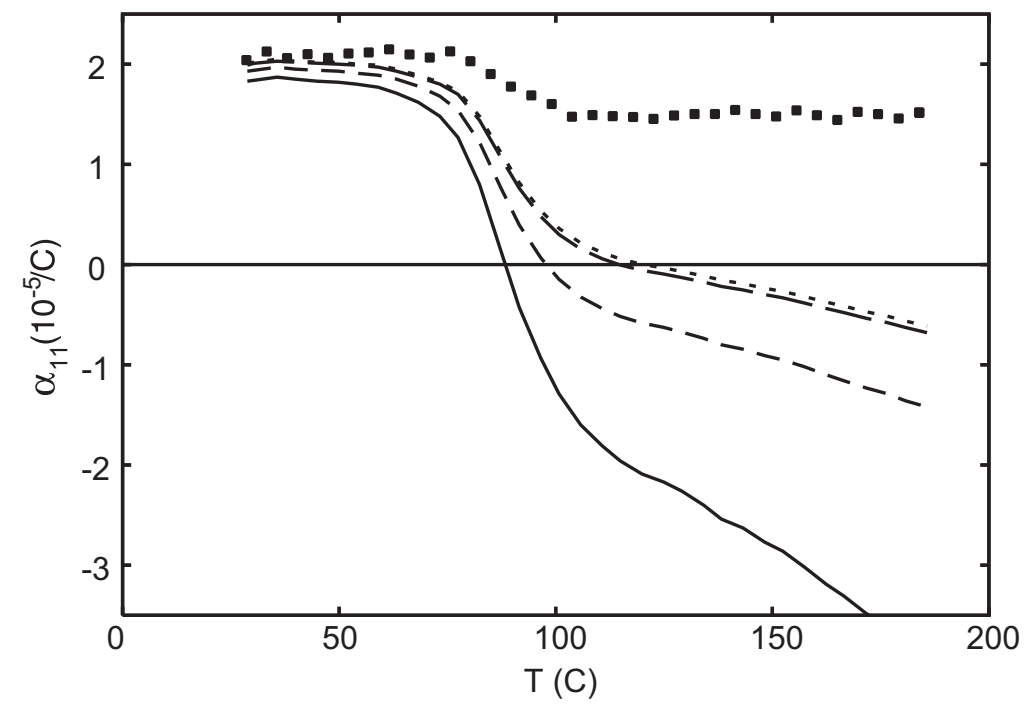

(b)

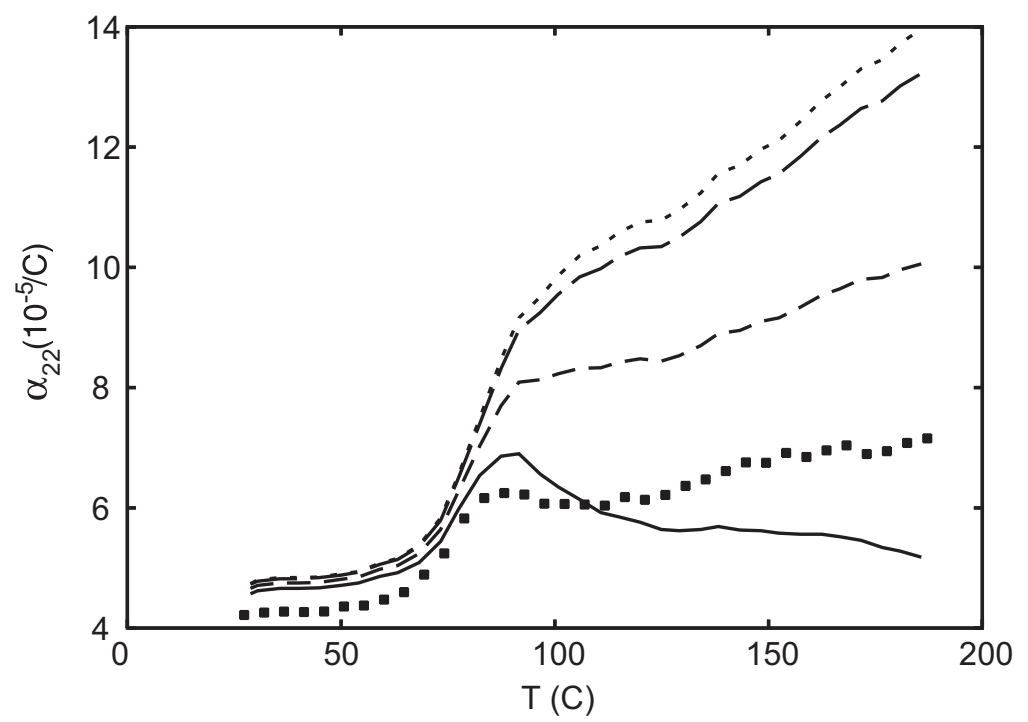

(c)

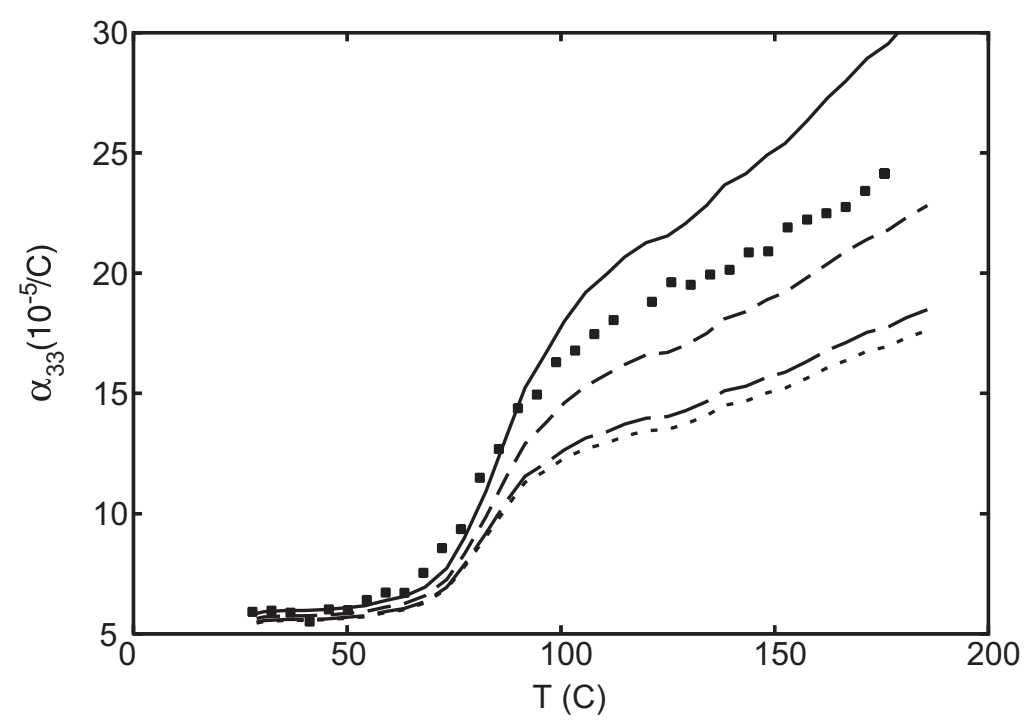


